\title{
Effective adoptive immunotherapy of triple- negative breast cancer by folate receptor- alpha redirected CAR T cells is influenced by surface antigen expression level
}

De-Gang Song ${ }^{1,4}$, Qunrui Ye ${ }^{1}$, Mathilde Poussin ${ }^{1}$, Jessica A. Chacon ${ }^{1}$, Mariangela Figini ${ }^{2}$ and Daniel J. Powell Jr. ${ }^{1,3^{*}}$

\begin{abstract}
Background: The poor prognosis and the limited efficacy of targeted therapy in patients with triple-negative breast cancer (TNBC) have raised the need for alternative therapies. Recent studies have demonstrated that folate receptor-alpha (FRa) may represent an ideal tumor-associated marker for immunotherapy for TNBC.

Methods: The aim of the present study was to apply a chimeric antigen receptor (CAR) approach for the targeting of FRa expressed on TNBC cells and evaluate the antitumor activity of CAR-engineered T cells in vitro and in vivo.

Results: We found that human T cells expressing a FRa-specific CAR were potent and specific killers of TNBC cells that express moderate levels of FRa in vitro and significantly inhibited tumor outgrowth following infusion into immunodeficient mice bearing an MDA-MB-231 tumor xenograft. However, the antitumor activity of the FRa CAR T cells was modest when compared to the same CAR T cells applied in an ovarian tumor xenograft model where FRa expression is more abundant. Notably, FRa CAR T cells induced superior tumor regression in vivo against MDA-MB-231 that was engineered for overexpression of FRa.

Conclusions: Taken together, our results show that FRa CAR T cells can mediate antitumor activity against established TNBC tumor, particularly when FRa is expressed at higher levels. These results have significant implications for the pre-selection of patients with high antigen expression levels when utilizing CAR-based adoptive $T$ cell therapies of cancer in future clinical trials.
\end{abstract}

Keywords: Folate receptor-alpha, Triple-negative breast cancer, Chimeric antigen receptor, Immunotherapy

\section{Background}

Triple-negative breast cancer (TNBC) is characterized by the limited expression of the human epidermal growth factor receptor 2 (HER2), estrogen receptor (ER), and progesterone receptor (PR) and accounts for approximately $15 \%$ of invasive breast cancers. Patients with TNBC do not benefit from HER2-based targeted therapy or endocrine therapy [1]. Chemotherapy is currently the mainstay

\footnotetext{
* Correspondence: poda@mail.med.upenn.edu

'Ovarian Cancer Research Center, Department of Obstetrics and Gynecology, Perelman School of Medicine, University of Pennsylvania, 3400 Civic Center Blvd, Rm 8-103 Smilow CTR, Philadelphia, PA 19104, USA

${ }^{3}$ Department of Pathology and Laboratory Medicine, Abramson Cancer Center, Perelman School of Medicine, University of Pennsylvania, 3400 Civic Center Blvd, Rm 8-103 Smilow CTR, Philadelphia, PA 19104, USA

Full list of author information is available at the end of the article
}

of systemic medical treatment, although patients with TNBC have a worse outcome after chemotherapy compared to breast cancer patients with other subtypes [2], a finding that reflects the intrinsically adverse prognosis associated with the disease. Thus, new and powerful therapies are urgently needed for TNBC patients.

A number of potential antigen targets have been validated in TNBC [3]. Folate receptor-alpha (FR $\alpha)$ is highly expressed in non-mucinous tumors of epithelial origin including ovarian, breast, and lung cancers and expressed at low levels on the apical surface of a subset of polarized epithelial cells including the parotid, kidney, lung, thyroid, and breast. Specific overexpression of FR $\alpha$ in certain malignancies, including TNBC [4], with low coordinate expression in normal tissue, makes $\mathrm{FR} \alpha$ an attractive target 
for directed therapies. In breast cancer, FR $\alpha$ expression can be regulated by steroid hormones, particularly estrogens $[5,6]$. Specifically, $17 \beta$-estradiol has been demonstrated to down-regulate FR $\alpha$ expression by direct action of the ER on the P4 promoter of FR $\alpha$, suggesting a negative correlation between the expression of ER and FR $\alpha$. Indeed, O'Shannessy et al. demonstrated that ER-negative breast cancer samples were significantly more likely to express FR $\alpha$ than ER-positive cancers. Taken together, these findings rationalize the assessment of FR $\alpha$ as a tumor-associated antigen candidate for the targeted therapy of TNBC.

Given its cancer-centric overexpression, FR $\alpha$ has been an attractive candidate for targeted drug delivery using folate-conjugated therapeutic compounds that bind FR $\alpha$ or murine, chimeric, and humanized monoclonal antibodies (mAbs) alone or in conjugates to deliver radionuclides, $\mathrm{T}$ cells, and stimulatory cytokines to malignant tissue $[7,8]$. Additionally, the transfer of T cells genetically redirected with a chimeric antigen receptor (CAR) specific for FR $\alpha$ is an attractive technology that is actively being investigated $[9,10]$. The CAR approach combines the antigen specificity of an antibody with the ability of T cells to mediate the killing of tumor cells in a single fusion molecule. CAR-modified $\mathrm{T}$ cells actively and specifically target their specified antigen and have the capacity to persist as memory cells in vivo $[9,10]$. As such, CAR-modified T cells that target tumor-associated antigens (TAAs), such as $F R \alpha$, may be more effective than mAbs in generating durable tumor responses. Here, we generated a FR $\alpha$ specific CAR with an intracellular CD27 co-stimulatory signaling domain and evaluated the therapeutic efficacy of $\mathrm{T}$ cells transduced to express this CAR in a murine xenograft model of human TNBC. We demonstrate that FR $\alpha-$ specific CAR $T$ cells have the capacity to inhibit human TNBC growth in vivo and that more robust tumor regression is achievable when the TNBC cells overexpress surface FR $\alpha$ protein.

\section{Methods}

\section{Cell lines}

Lentivirus packaging was executed using the immortalized normal fetal renal 293T cell line purchased from ATCC. Human cell lines used in immune-based assays include the established human ovarian cancer cell lines SKOV3 and C30 and breast cancer cell lines T47D, SKBR3, MCF7, MDA-231, MDA-436, MDA-468, MDA-453, and BT549. For bioluminescence assays, the cancer cell lines were transfected to express firefly luciferase (fLuc). The mouse malignant mesothelioma cell line, AE17 (kindly provided by Steven Albelda, University of Pennsylvania), was used as antigen negative control. All cell lines were maintained in R10 medium: RPMI-1640 supplemented with $10 \%$ heat-inactivated FBS, $100 \mathrm{U} / \mathrm{mL}$ penicillin, $100 \mathrm{mg} / \mathrm{mL}$ streptomycin sulfate, and $10 \mathrm{mmol} / \mathrm{L}$ HEPES.

\section{CAR construction and lentivirus production}

The anti-FR $\alpha$ CAR construct was comprised of the MOv19 scFv linked to a CD8a hinge and transmembrane region, followed by a CD3z signaling moiety in tandem with the CD27 intracellular signaling motif (MOv19-27z; Fig. 1a) and was previously described $[9,10]$. An anti-CD19 CAR containing CD3z and CD27 signaling motifs in tandem $(\mathrm{CD} 19-27 \mathrm{z})$ was used as an antigen specificity control $[9,11]$. High-titer replication-defective lentiviruses were produced and concentrated as previously described [12]. Briefly, $293 \mathrm{~T}$ cells were seeded in $150-\mathrm{cm}^{2}$ flask and transfected using Express In (Open Biosystems) according to the manufacturer's instructions. FR $\alpha$-specific CAR transgene plasmids $(15 \mu \mathrm{g})$ were co-transfected with $18 \mu \mathrm{g}$ pRSV.REV (Rev expression plasmid), $18 \mu \mathrm{g}$ pMDLg/p.RRE (Gag/Pol expression plasmid), and $7 \mu \mathrm{g}$ pVSV-G (VSV glycoprotein expression plasmid) with $174 \mu \mathrm{L}$ Express In $(1 \mu \mathrm{g} / \mu \mathrm{L})$ per flask. Supernatants were collected at 24 and $48 \mathrm{~h}$ after transfection, concentrated 10 -fold by ultracentrifugation for $2 \mathrm{~h}$ at 28,000 rpm with a Beckman SW32Ti rotor (Beckman Coulter). The viruses were aliquoted into tubes and stored at $-80{ }^{\circ} \mathrm{C}$ until ready to use for titering or experiments. All lentiviruses used in the experiments were from concentrated stocks.

\section{Human $T$ cells and transfection}

Primary human $\mathrm{T}$ cells, purchased from the Human Immunology Core at University of Pennsylvania, were isolated from healthy, normal donors following leukapheresis by negative selection. All $\mathrm{T}$ cell samples were collected under a protocol approved by a University Institutional Review Board, and written informed consent was obtained from each healthy, normal donor. $\mathrm{T}$ cells were cultured in R10 medium and stimulated with anti-CD3 and anti$\mathrm{CD} 28$ monoclonal antibody (mAb)-coated beads (Invitrogen). Approximately 18 to $24 \mathrm{~h}$ after activation, human $\mathrm{T}$ cells were transduced using a spinoculation procedure. Briefly, $0.5 \times 10^{6} \mathrm{~T}$ cells were infected with a multiplicity of infection (MOI) of 5 of the MOv19-27z vector. A mixture of cells and vectors were centrifuged at room temperature for $90 \mathrm{~min}(2500 \mathrm{rpm})$ in a table-top centrifuge (Sorvall ST 40). After the engineered T cells were rested, as determined by decreased growth kinetics and cell size which is measured using the Multisizer 3 Coulter Counter (Beckman Coulter), the rested $\mathrm{T}$ cells were then used for functional analysis.

\section{Flow cytometric analysis}

The following fluorochrome-conjugated mAbs, purchased from BD Biosciences, were used for phenotypic analysis: APC-Cy7 anti-human CD3, fluorescein isothiocyanate 

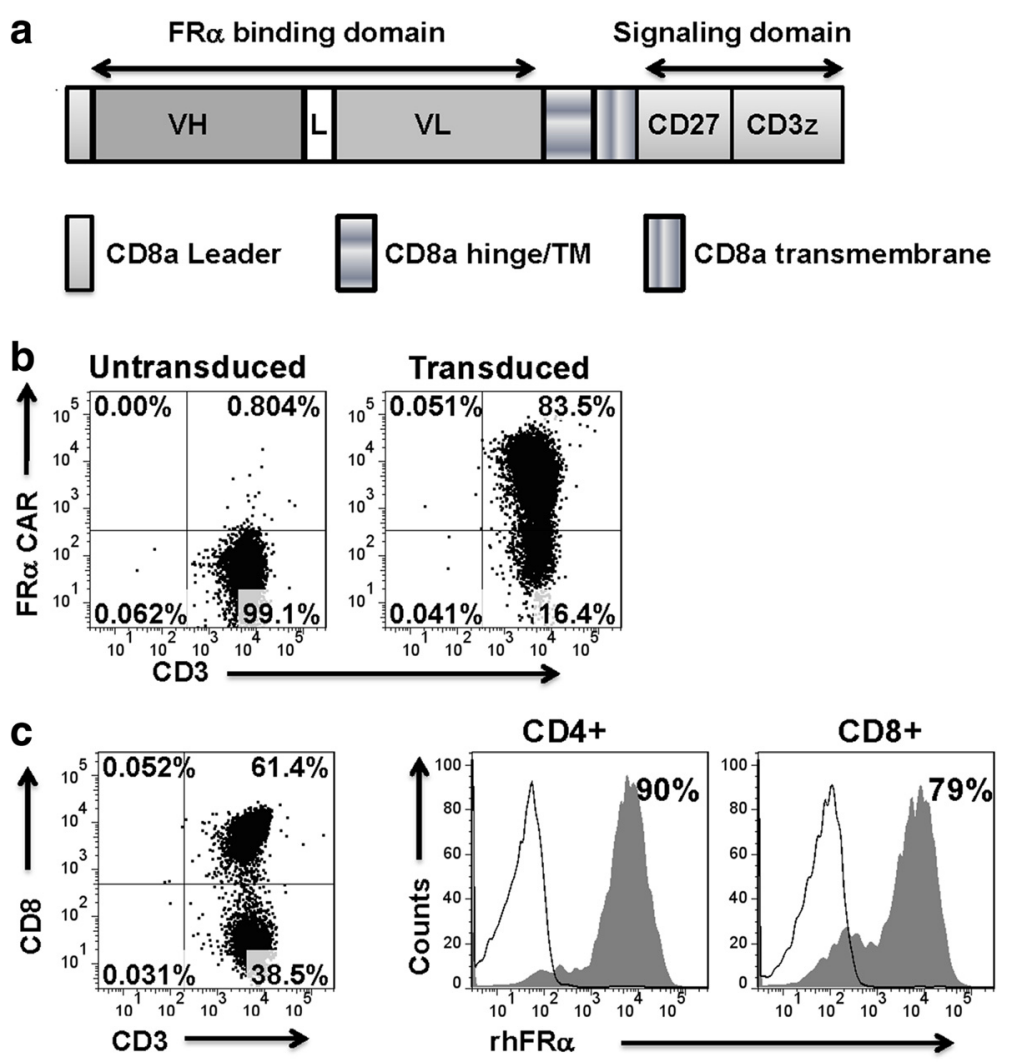

Fig. 1 Construction and expression of folate receptor-alpha (FRa)-specific chimeric antigen receptor (CAR). a Schematic representation of MOv19based FRa CAR constructs containing the CD27 co-stimulatory module in combination with the CD3 cytosolic domain. b Primary human CD3 T cells can efficiently express FRa-specific CAR. Expression was detected via PE-conjugated goat anti-mouse F(ab') ${ }_{2}$ fragment and measured by flow cytometry. c Compared to untransduced (UNT) T cells, transduced T cells consisted of CD4- and CD8-positive cells with both subsets expressing FRa CAR. FRa CAR expression was detected via biotin-labeled recombinant FRa protein staining followed by streptavidin-PE after transduction with lentivirus. Transduction efficiencies are indicated with the percentage of CAR expression in parentheses

(FITC) anti-human CD4, APC anti-human CD8, PEanti-human CD45, and PE anti-human CD137. 7-Aminoactinomycin D (7-AAD) was used for viability staining. For the in vivo mouse $\mathrm{T}$ cell transfer experiments, peripheral blood was obtained via retro-orbital bleeding and stained for the presence of human CD45, CD4, and CD8 $T$ cells. Gating specifically on the human CD45+ population, the CD4+ and CD8+ subsets were quantified using TruCount tubes (BD Biosciences) with known numbers of fluorescent beads as described in the manufacturer's instructions. Tumor cell lines and enzymatically digested MDA-231 tumor cell surface expression of FR $\alpha$ was measured using human PE-FR $\alpha$ mAb (R\&D Systems, Inc.) and Quanti-Brite PE beads (BD Bioscience). The FR $\alpha$ PEconjugated antibody was used at a 1:1 PE/protein ratio for the quantitative analysis of surface FR $\alpha$ expression. FR $\alpha$ antigen copy number per tumor cell was evaluated by comparing the mean fluorescence intensity (MFI) versus the number of known PE molecules per bead. A calibration curve was constructed per cell line to calculate the mean number of PE molecules per cell for the FR $\alpha+$ cell population. Mouse mesothelioma cell line AE17 was used as a negative control, and the background human FR $\alpha$ receptors/cell values measured on AE17 were subtracted from the FR $\alpha$ receptors/cell values detected on breast cancer cell lines.

FR $\alpha$ CAR surface expression was evaluated using recombinant FR $\alpha$-Fc protein (R\&D Systems) followed by PE-labeled anti-human IgG Fc gamma-specific antibody (eBioscience) or biotin-labeled goat anti-mouse IgG $\mathrm{F}\left(\mathrm{ab}^{\prime}\right)_{2}$ fragment followed by streptavidin-APC. For intracellular cytokine staining, $\mathrm{T}$ cells were stimulated in R10 media containing phosphomolybdic acid (PMA) (30 ng/mL) (Sigma-Aldrich), ionomycin $(500 \mathrm{ng} / \mathrm{mL}$ ) (Sigma-Aldrich), and monensin (GolgiStop) $(1 \mu \mathrm{L} / \mathrm{mL})$ (BD Biosciences) in a cell incubator with $10 \% \mathrm{CO}_{2}$ at $37{ }^{\circ} \mathrm{C}$ for $4 \mathrm{~h}$. Cytokine production in CAR T cells was determined by co-culturing CAR $\mathrm{T}$ cells with $\mathrm{FR}^{\mathrm{pos}}$ ovarian cancer cells for $5 \mathrm{~h}$. After the cell surface markers were stained, the cells were then fixed and permeabilized using Cytofix/ Cytoperm and Perm/Wash buffer (eBioscience) according to the manufacturer's instructions. The cells were then 
stained with the following fluorescence-conjugated cytokine antibodies: PE anti-human interferon-gamma (IFN- $\gamma$ ), Pacific Blue anti-human TNF- $\alpha$, and FITC anti-human IL-2. All flow cytometry was conducted using a BD FACSCanto II flow cytometer (BD Biosciences), and flow cytometric data were analyzed with Flowjo version 7.6.1 software (Tree Star, Ashland, OR).

\section{Cytokine release assays}

Cytokine release assays were performed by co-culturing $1 \times 10^{5} \mathrm{~T}$ cells with $1 \times 10^{5}$ target cells in triplicate in a 96-well flat bottom plate in a total volume of $200 \mu \mathrm{L}$ R10 media. After 20-24 h, co-culture supernatants were collected and ELISA (Biolegend, San Diego) was performed, according to the manufacturer's instructions, to measure the secretion of IFN- $\gamma$. The values shown represent the mean of triplicate wells.

\section{Cytotoxicity assays}

For cell-based bioluminescence assays, $5 \times 10^{4}$ firefly luciferase (fLuc)-expressing tumor cells were cultured with R10 media in the presence of different $\mathrm{T}$ cell ratios in a 96-well Microplate (BD Biosciences). After incubation for $\sim 20 \mathrm{~h}$ at $37^{\circ} \mathrm{C}$, each well was filled with $50 \mu \mathrm{L}$ of DPBS resuspended with $1 \mu \mathrm{L}$ of D-luciferin $(0.015 \mathrm{~g} / \mathrm{mL})$ and imaged with the Xenogen IVIS Spectrum. Tumor cell viability percentage was calculated as the mean luminescence of the experimental sample minus background divided by the mean luminescence of the input number of target cells used in the assay minus background times 100 . All data are represented as a mean of triplicate wells.

\section{Xenograft model of TNBC and ovarian cancer}

All animals were obtained from the Stem Cell and Xenograft Core of the Abramson Cancer Center, University of Pennsylvania. NOD/SCID/ $\gamma$-chain ${ }^{-/-}$(NSG) mice (6-12 weeks old) were bred, treated, and maintained under pathogen-free conditions in-house under University of Pennsylvania IACUC-approved protocols. To establish a TNBC model, 6- to 12-week-old female NSG mice were inoculated subcutaneously (s.c.) on the flank with $3 \times 10^{6}$ MDA-231 fLuc+ or MDA-231.FR fLuc cells on day 0. For the ovarian cancer model, NSG mice were inoculated s.c. on the flank with $5 \times 10^{6}$ SKOV3 fLuc+ cells. After the tumors become palpable at about 3 weeks, primary human $\mathrm{T}$ cells were activated and transduced as described above. After the primary human T cells were expanded for 2 weeks and the mouse tumor burden was about $200-300 \mathrm{~mm}^{3}$, the mice were treated with the T cells. The route, dose, and timing of $\mathrm{T}$ cell injections are indicated in the individual figure legends. Tumor dimensions were measured with calipers and tumor volumes calculated using the formula $V=1 / 2$ (length $\times$ width $^{2}$ ), where length is the greatest longitudinal diameter and width is the greatest transverse diameter. Animals were imaged prior to T cell transfer and about every week thereafter to evaluate tumor growth. Photon emission from fluc+ cells was quantified using the "Living Image" software (Xenogen) for all in vivo experiments. Approximately 40 days after the first $\mathrm{T}$ cell injection, the mice were euthanized and the tumors were resected immediately in order to calculate the tumor volumes.

\section{Bioluminescence imaging}

Tumor growth was also monitored using bioluminescence imaging (BLI). BLI was conducted using Xenogen IVIS imaging system. The photons emitted from fluc-expressing cells within the animal body were quantified using Living Image software (Xenogen). Briefly, mice bearing MDA-231 fLuc+ or MDA-231.FR fLuc+ tumor cells were injected intraperitoneally (i.p.) with D-luciferin $(150 \mathrm{mg} / \mathrm{kg}$ stock, $100 \mu \mathrm{L}$ of D-luciferin per $10 \mathrm{~g}$ of mouse body weight) suspended in PBS and imaged under isoflurane anesthesia after 5 10 min. A pseudocolor image representing light intensity (blue, least intense; red, most intense) was generated using Living Image. BLI findings were confirmed at necropsy.

\section{Statistical analysis}

The data are reported as means and standard deviations (SDs). Statistical analysis was performed using two-way repeated-measure analysis of variance (ANOVA) for the tumor burden (tumor volume, photon counts). Student's $t$ test was used to evaluate differences in absolute numbers of transferred $\mathrm{T}$ cells, cytokine secretion, and specific cytolysis. GraphPad Prism 5.0 (GraphPad Software) was used for the statistical calculations, where a $p$ value of $p<0.05$ was considered significant.

\section{Results}

\section{Construction and expression of FRa-specific CAR}

A FR $\alpha$-specific CAR-encoding lentivirus construct was generated comprised of the anti-human FR $\alpha$-specific MOv19 scFv [13] linked to a CD8 $\alpha$ hinge and transmembrane region, followed by a CD27 intracellular signaling motif in tandem with the $\mathrm{CD} 3 \zeta$ signaling moiety, and referred to as MOv19-27z (Fig. 1a). Primary human $\mathrm{CD} 3+\mathrm{T}$ cells were efficiently transduced with CAR lentiviral vectors with reproducible transduction efficiencies of $\sim 80 \%$ (Fig. 1b). Surface expression of the FR $\alpha$ CAR on $\mathrm{CD} 4+$ and $\mathrm{CD} 8+\mathrm{T}$ cells was detectable using recombinant FR $\alpha$ protein staining (Fig. 1c), demonstrating positive CAR expression and antigen binding capacity.

\section{FRa is expressed on the surface of TNBC cell lines}

Using flow cytometry, surface expression of FR $\alpha$ protein was determined on a variety of tumor cell lines after staining the cells with anti-FR $\alpha$ antibody. FR $\alpha$ expression 
was detected in ovarian cancer lines SKOV3 and A1847 at high levels and also on the breast cancer cell lines, T47D, SKBR3, and MCF7; the C30 ovarian cancer cell line served as an antigen negative control (Fig. 2). Importantly, all five TNBC cell lines tested, including MDA-231, MDA-468, MDA-436, MDA-453, and BT549, expressed FR $\alpha$ protein on their cell surface at moderate to low levels (Fig. 2). As demonstrated in Additional file 1: Figure S1A, there was a range of FR $\alpha$ molecules expressed per cell among the breast cancer cell lines tested.

\section{FRa CAR T cells specifically recognize FRa ${ }^{\text {pos }}$ TNBC cell lines in vitro}

To determine whether human FR $\alpha$ CAR-modified T cells were able to recognize and react against $\mathrm{FR}^{\mathrm{pos}} \mathrm{TNBC}$ cells, FR $\alpha$ CAR-bearing $\mathrm{T}$ cells were co-cultured overnight with the TNBC cell lines MDA-231, MDA-468, MDA-436, MDA-453, or BT549, and IFN- $\gamma$ secretion in the cell culture supernatants was measured by ELISA. Since ovarian cancers and breast cancers frequently express FR $\alpha$, established human ovarian cancer cell lines (SKOV3) and breast cancer cell lines (T47D, SKBR3, and MCF7) that express surface FR $\alpha$ at varying levels were used for positive control targets, while FR $\alpha$-negative cell lines C30 and AE17 were used as negative controls. As shown in Fig. 3a, FR $\alpha$ CAR T cells secreted substantial amounts of IFN- $\gamma$, denoting T cell activation after co-culture with $\mathrm{FR}^{\mathrm{pos}} \mathrm{TNBC}$ cell lines. The number of FR $\alpha$ molecules/cells correlated with the amount of IFN- $\gamma$ secreted (Additional file 1: Figure S1B) and determined the coefficient of determination value $\left(R^{2}=0.62\right)$. No IFN- $\gamma$ production was detected when FR $\alpha$ CAR $\mathrm{T}$ cells were cultured with FR $\alpha$-negative targets (C30 and AE17) or from co-cultures containing untransduced (UNT) T cells. Up-regulated CD137 (4-1BB) expression represents a surrogate marker for antigenspecific activation of functional human CD8+ T cells [14]. When FR $\alpha$ CAR $T$ cells were co-cultured with $\mathrm{FR}^{\mathrm{pos}}$ or $\mathrm{FR} \alpha^{\text {neg }}$ tumor cells, robust up-regulation of CD137 by CAR $\mathrm{T}$ cells was observed only when incubated with $\mathrm{FR}^{\mathrm{pos}}$ TNBC cells (Fig. 3b). CD137 up-regulation was not detected on anti-CD19 CAR T cells when similarly cultured with $\mathrm{FR}^{\mathrm{pos}} \mathrm{TNBC}$ cells, indicating that $\mathrm{CD} 137$ up-regulation by FR $\alpha$ CAR T cells was antigen-specific.

In addition to the above assays, representative fluorescence-activated cell sorter (FACS) plots of 5-h intracellular expression of proinflammatory cytokines by FR $\alpha$ CAR $T$ cells in response to FR $\alpha^{\text {pos }}$ TNBC cells are shown (Fig. 3c). Th1 cytokines including IFN- $\gamma$, TNF- $\alpha$, and IL-2 were exclusively expressed in FR $\alpha$ CAR T cells and not in UNT control T cells, when incubated with the $\mathrm{FR}^{\mathrm{pos}}$ MDA-231 TNBC cell line. PMA/ionomycintreated $\mathrm{T}$ cells served as positive controls for $\mathrm{T}$ cellstimulated cytokine production.

\section{FRa CAR T cells have antitumor activity against MDA-231} in vitro and in vivo

The cytolytic activity of FR $\alpha$ CAR T cells in vitro was evaluated using an overnight bioluminescence assay (Fig. 4a). FR $\alpha$ CAR T cells had robust and specific cytotoxic activity against FR $\alpha^{\text {pos }}$ MDA-231 cells but not FR $\alpha$-negative C30 cells. Untransduced or control anti-CD19 CAR T cells did not lyse MDA-231 or C30 cell lines.

To assess the antitumor activity of CAR T cells targeting $\mathrm{FR} \alpha^{\mathrm{pos}}$ tumor in vivo, we first evaluated the potency

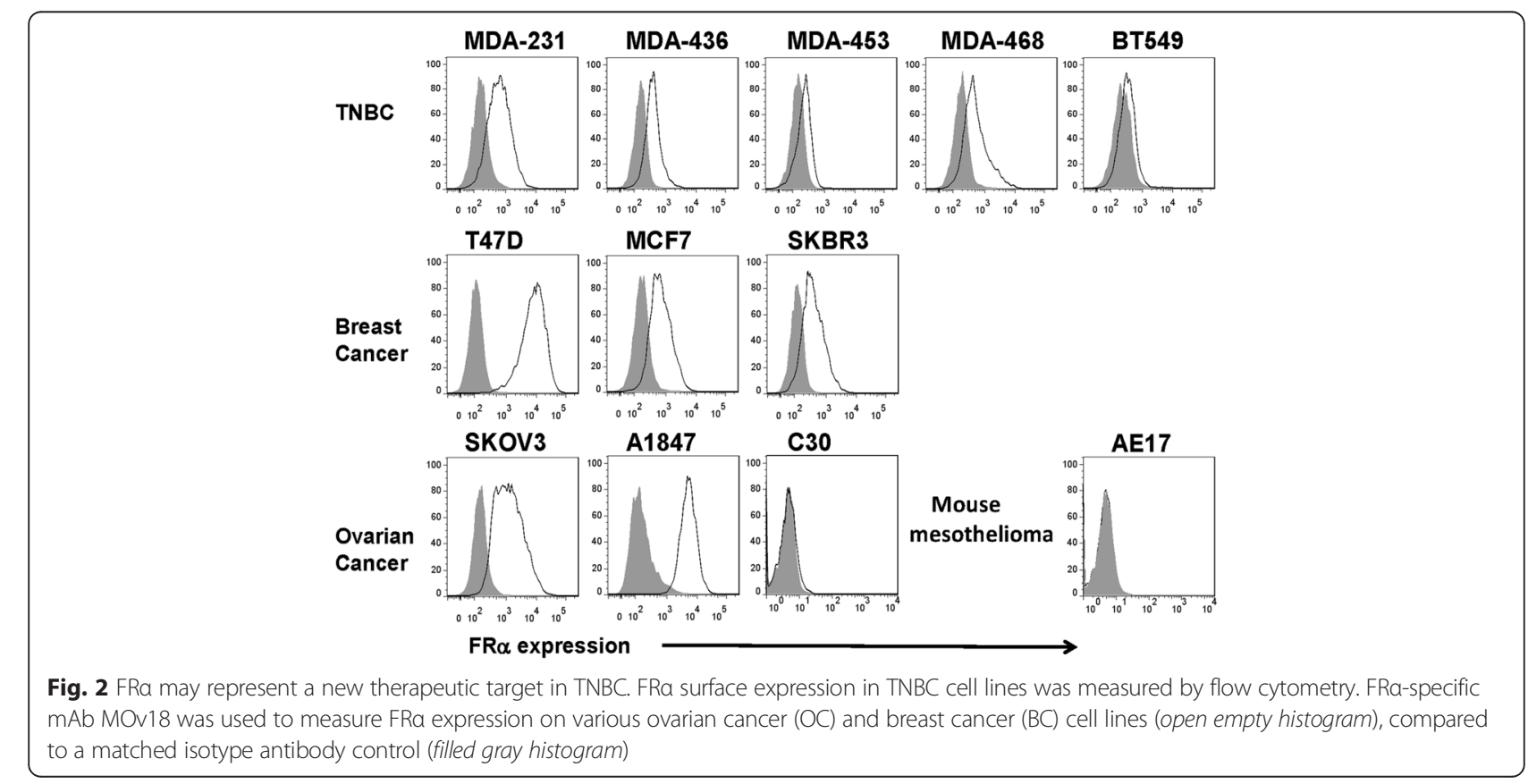




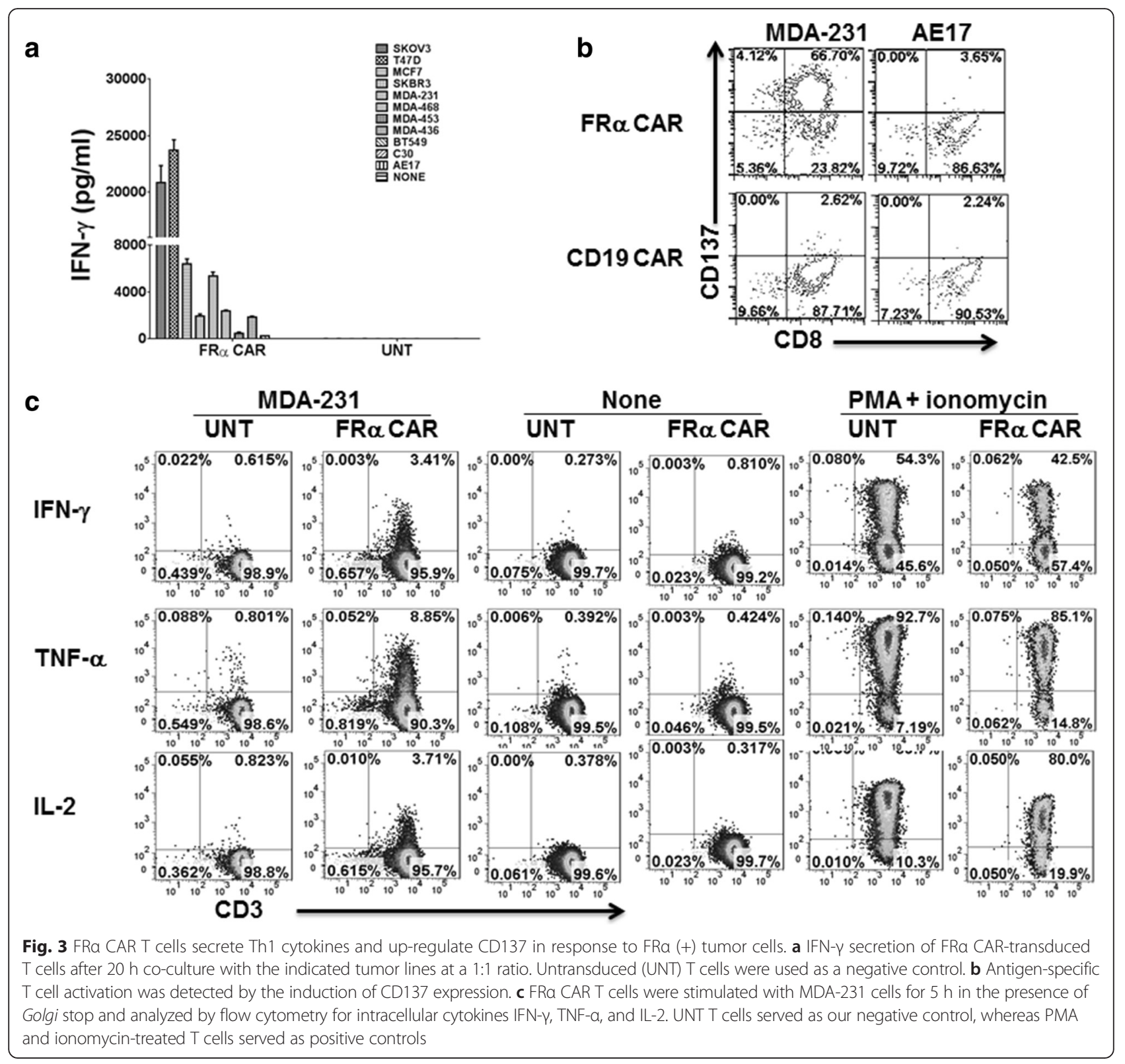

of FR $\alpha$ CAR T cells using a xenograft mouse model of TNBC tumor. Immunodeficient non-obese diabetic/severe combined immunodeficient/IL-2 $\gamma \mathrm{c}^{\text {null }}$ (NSG) mice received subcutaneous inoculation of firefly luciferase (fLuc+) $\mathrm{FR}^{\text {pos }}$ human TNBC MDA-231 cells on the hind flank and received intravenous (I.V.) injections of $10^{7}$ CAR+ T cells on days 40 and 46 after tumor inoculation, when tumors were $\sim 250 \mathrm{~mm}^{3}$ in size and evident by bioluminescence imaging (BLI). Infused FR $\alpha$-specific MOv19-27z CAR T cells mediated significant, albeit modest, reduction in tumor progression compared to the control mice treated with untransduced T cells $(p=0.01)$ or with anti-CD19 CAR T cells $(p=0.035)$, as measured by caliper-based tumor size (Fig. 4b).
We next measured the persistence of the transferred human $T$ cells in vivo to determine whether modest antitumor activity was associated with poor T cell engraftment. Peripheral blood was obtained from MDA-231-bearing NSG mice treated with IV injections of T cells on day 60, 14 days after the last dose of transferred $\mathrm{T}$ cells, and quantified for the presence of human CD4+ and CD8+ T cells. Mice that received FR $\alpha$-specific MOv19-27z T cells had readily detectable circulating human CD4+ and CD8+ T cells with significantly higher cell counts than those observed in mice treated with anti-CD19 CAR or untransduced T cells (Fig. 4c; $p=0.008$ and $p=0.002$, respectively), indicating that tumor antigen recognition drives the survival of the adoptively transferred FR $\alpha$-specific CAR T cells in vivo. 

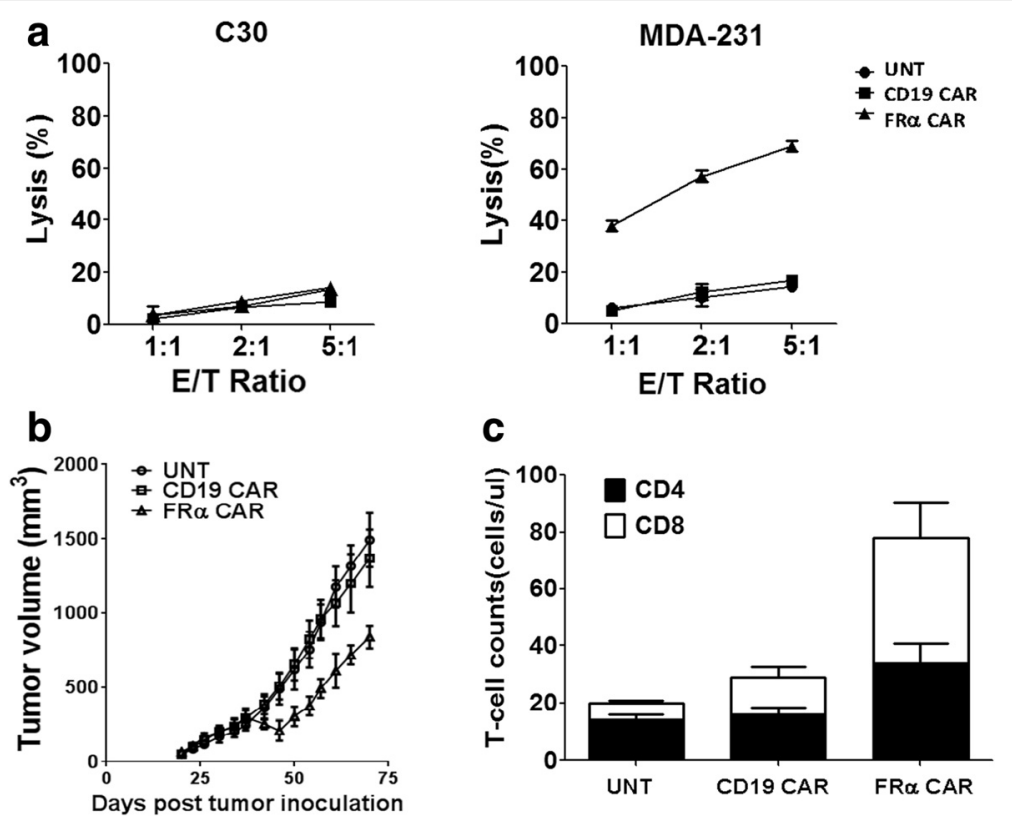

Fig. 4 Anti-tumor activity of FRa CAR T cells in vitro and in vivo. a FRa CAR T cells lysed FRa+ MDA-231 cells but exhibited decreased lysis of the FRa-C30 cells at the indicated effector/target (E/T) ratio for $\sim 20 \mathrm{~h}$. Untransduced (UNT) T cells served as our negative control. b NSG mice bearing established subcutaneous (s.c.) tumor were treated with intravenous (i.v.) injections of $1 \times 10^{7}$ CAR+ T cells on days 40 and 46 post tumor inoculation. Tumor growth was assessed by caliper measurement $\left[V=1 / 2\left(\right.\right.$ length $\times$ width $\left.\left.^{2}\right)\right]$. c Peripheral blood was collected 3 weeks after the first T cell infusion and quantified for the absolute number of human CD4+ and CD8+T cells/ $\mu \mathrm{L}$ of blood. Mean cell count \pm SEM is shown with $n=5$ for all groups

We next assayed for FR $\alpha$ protein expression on residual tumors after treatment with untransduced, anti-CD19 CAR or FR $\alpha$-specific CAR T cells, to determine whether tumor outgrowth in FR $\alpha$ CAR-treated mice was a byproduct of immune pressure and selective growth of FR $\alpha$ negative cancer cells. MDA-231 tumors retained a stable FR $\alpha$ expression profile (Additional file 2: Figure S2), indicating that antigen loss did not occur after CAR $\mathrm{T}$ cell treatment in this TNBC xenograft model.

\section{FRa CAR T cells preferentially kill antigen overexpressing tumor cells}

We previously showed that FR $\alpha$ CAR T cells can eradicate $\mathrm{FR} \alpha^{\mathrm{pos}}$ ovarian cancer xenografts in vivo $[9,10]$ and that FR $\alpha$ expression on MDA-231 cells is sufficient for recognition by FR $\alpha$ CAR T cells in vitro and in vivo. In parallel assays, we observed superior tumor regression in a FR $\alpha^{\text {pos }}$ SKOV3 ovarian cancer xenograft model where mice were treated with the same FR $\alpha$ CAR T cells (Additional file 3: Figure S3), reflecting a disparity in the killing of TNBC MDA-231 tumors versus $\mathrm{FR} \alpha^{\mathrm{pos}} \mathrm{SKOV} 3$ ovarian tumors in vivo. We postulated that this resulted from the higher relative expression of FR $\alpha$ protein by SKOV3 than MDA231, which reduced the potential of CAR T cells to exert their full function against TNBC in vivo and that TNBC cells with higher FR $\alpha$ expression might be more responsive to therapy. To test this hypothesis, MDA-231 cells were engineered to overexpress human FR $\alpha$ (referred to as MDA-231.FR $\alpha$ ). The MDA-231.FR $\alpha$ cell line exhibited a 19-fold increase in mean fluorescence intensity for FR $\alpha$ expression compared to the parental line (Fig. 5a). In an in vitro cytotoxicity assay, FR $\alpha$ CAR T cells were capable of killing MDA-231.FR $\alpha$ cells as well as SKOV3 cells and more efficiently than parental MDA-231 cells (Fig. 5b). Untransduced T cells served as a negative control and did not recognize or kill tumor cells despite the high levels of FR $\alpha$ expression. Thus, it appears that tumor cells expressing antigen at low levels are less sensitive to killing by CAR T cells. We therefore sought to determine whether CAR $\mathrm{T}$ cells preferentially kill tumor cells that express antigen at higher levels and selectively spare tumor cells with lower FR $\alpha$ levels. We first engineered parental MDA231 cells that express moderate levels of FR $\alpha$ to express green fluorescent protein (GFP), referred to as MDA231.GFP, and then co-cultured MDA-231.FR $\alpha$ (GFP-negative) cells with MDA-231.GFP cells at a 1:1 ratio, where $\sim 50 \%$ of all MDA-231 cells were found to express GFP (Fig. 5c). The 1:1 MDA-231 mixture was then exposed to either untransduced or FR $\alpha$-specific CAR T cells at a target to effector cell ratio of 1:3 for $24 \mathrm{~h}$. Treatment with $\mathrm{FR} \alpha \mathrm{CAR} \mathrm{T}$ cells resulted in a relative increase in the number of MDA-231.GFP-engineered tumor cells ( 85 \% $\left.\mathrm{GFP}^{\mathrm{pos}}\right)$, reflecting a selective killing of the GFP-negative, FR $\alpha$ overexpressing MDA-231.FR $\alpha$ cell population (Fig. 5c). 


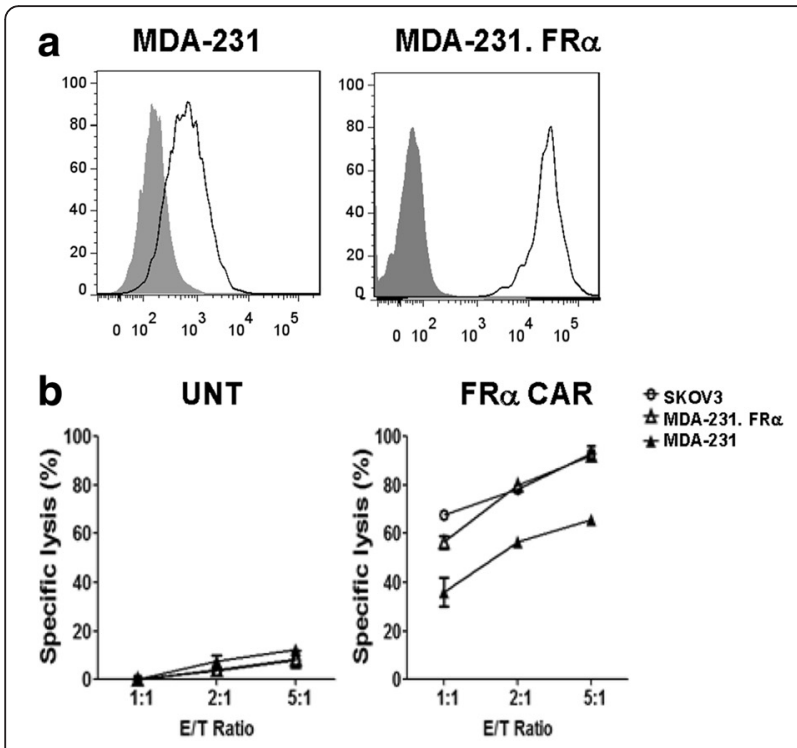

C MDA-231.FR $\alpha$ MDA-231.GFP 1:1 MIX

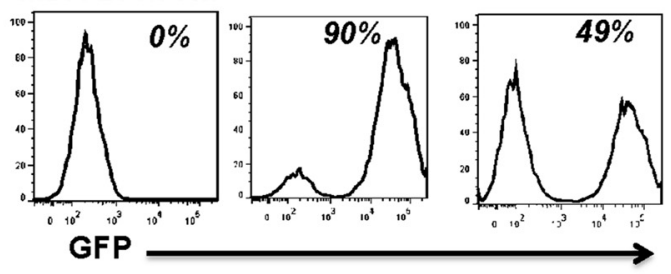
1:1 Mix plus

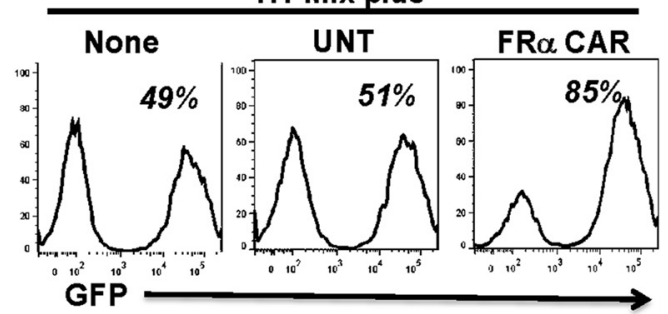

Fig. 5 FRa CAR T cells preferentially lyse tumor cells overexpressing FRa. a The MDA-231 cell line was engineered to overexpress FRa (MDA-231.FRa). b FRa CAR T cells T cells lyse MDA-231.FRa and SKOV3 more efficiently compared to MDA-231 cells. c MDA-231 cells were engineered to express $\sim 90 \%$ GFP (MDA-231.GFP). MDA-231.GFP cells were then mixed with MDA-231.FRa cells at a 1:1 ratio ( $50 \%$ GFP expression). The mixed cells were treated with either UNT or FRa CAR $T$ cells at a 3:1 of E/T ratio for $24 \mathrm{~h}$. UNT T cell treatment had no impact on the mixed tumor cell ( $\sim 50 \%$ GFP expression), whereas treatment with FRa CAR T cells increased the number of GFP (+)-engineered tumor cells ( $>5 \%$ GFP expression)

As anticipated, untransduced $\mathrm{T}$ cell treatment had no impact when co-cultured with tumor cells with $\sim 50 \%$ of MDA-231 cells still expressing GFP. These results suggest that tumor cells expressing higher antigen levels are preferentially killed, relative to those with lower target antigen expression.
FRa CAR T cells induce rapid tumor regression of TNBC overexpressing $F$ Ra in vivo

To evaluate the impact of the antigen level on antitumor activity of FR $\alpha$ CAR T cells in vivo, we inoculated NSG mice with MDA-231 or MDA-231.FR $\alpha$ cells and allowed for tumor growth. After 40 days, MDA-231.FR $\alpha$ tumors were modestly larger than their parental MDA-231 cells in mice, analogous to the known association between FR $\alpha$ overexpression and tumor progression [15] and the reported impact of FR $\alpha$ overexpression on ovarian cancer cell proliferation, migration, and invasion [15]. Micebearing established MDA-231.FR $\alpha$ or parental MDA-231 tumors received tail vein injections of $10^{7} \mathrm{CAR}+\mathrm{T}$ cells on days 40 and 46, and tumor growth was monitored using caliper measurements and BLI. Consistent with our initial in vivo assays, FR $\alpha$ CAR T cells modestly delayed MDA-231 tumor growth (Fig. 6a, b). However, the same dose of FR $\alpha$ CAR T cells mediated more effective tumor regression in mice with MDA-231.FR $\alpha$ tumors, despite larger initial tumor burden (Fig. 6c, d). On days 60 and 74 after tumor inoculation, the average MDA231.FR $\alpha$ tumor volume had decreased by 36 and $58 \%$, respectively, while the average MDA-231 tumor volume increased by 107 and $214 \%$, respectively (Additional file 4: Figure S4). By comparison, tumor volume increased sharply after treatment with anti-CD19 CAR T cells (Additional file 4: Figure S4). BLI confirmed that after treatment with FR $\alpha$ CAR T cells, mice bearing MDA-231.FR $\alpha$ tumors had less residual tumor burden, compared to mice with MDA-231 tumor (Fig. 6b, d), despite beginning therapy with greater tumor burden (Fig. 6b, d). Anti-CD19 CAR $T$ cells had no antitumor activity against MDA-231 or MDA-231.FR $\alpha$ tumors in vivo (Fig. 6a, c). These results suggest that the regression of TNBC mediated by CAR T cells is dependent on a sufficient level of surface tumor antigen expression.

\section{Discussion}

Although triple-negative breast cancer (TNBC) comprises approximately $15 \%$ of all breast cancer subtypes, its aggressive biology and lack of specific systemic regimen or targeted therapy mandates the search for novel treatments specific for TNBC. Folate receptor-alpha (FR $\alpha$; FOLR1) is a glycosyl-phosphatidyl inositol (GPI)-anchored protein that is overexpressed at both the protein and mRNA levels in TNBC [16], where it serves a biological role in TNBC cell growth and folate uptake. Strong FR $\alpha$ immunohistochemical (IHC) staining is highly associated with poor outcome in breast cancer patients [17], and while approximately $30 \%$ of breast cancers express FR $\alpha, 70-80 \%$ of stage IV metastatic TNBC tumors express FR $\alpha$ across multiple subtypes [4]. Importantly, the increased expression of FR $\alpha$ is associated with a significantly worse clinical outcome in TNBC [18]. Thus, the rationale for targeting 

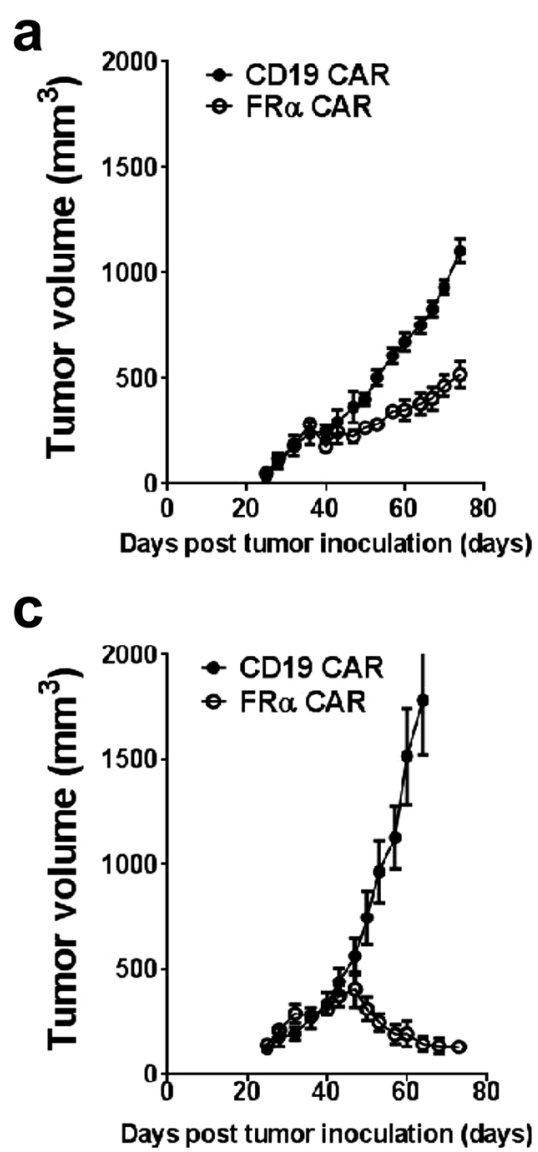

b

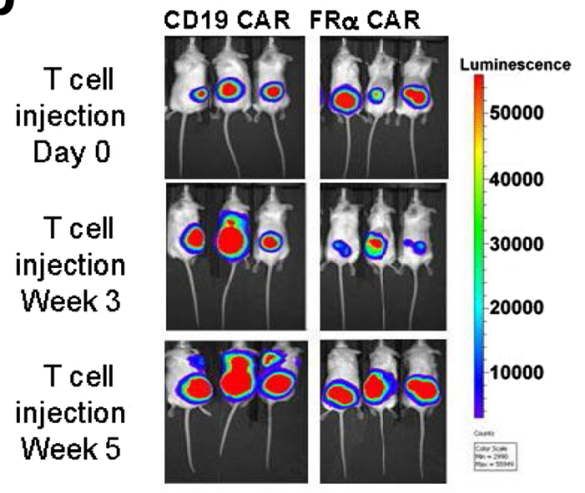

d

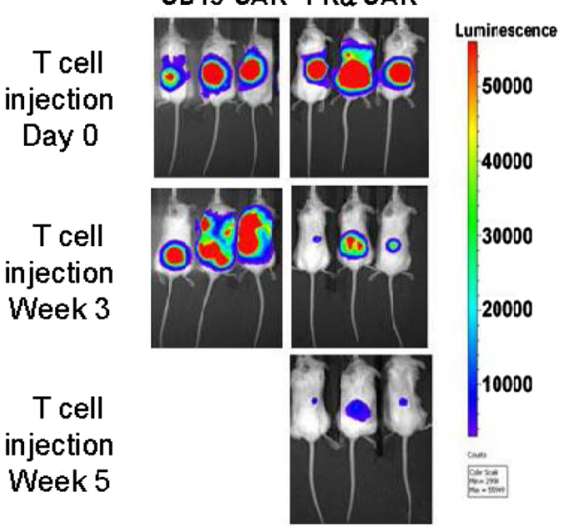

Fig. 6 FRa CART cells induce rapid tumor regression of MDA-231.FRa in vivo. a-d NSG mice were inoculated with MDA-231 (a, b) or MDA.231.FRa tumor cells (c, d). Mice bearing established MDA-231.FRa or MDA-231 tumors received tail vein injections of $1 \times 10^{7}$ CAR+ T cells on days 40 and 46 , and tumor growth was monitored by caliper measurements $(\mathbf{a}, \mathbf{c})$ and BLI (b, d)

FR $\alpha$ in TNBC is sound. Interestingly, endogenous immune responses against FR $\alpha$ are evident in cancer patients. For instance, Knutson et al. [19] demonstrated that immunity to $\mathrm{FR} \alpha$ is prevalent in patients with breast and ovarian cancers where $\mathrm{FR} \alpha$-reactive lymphocytes having been identified in patients with these cancers and attempts to bolster this natural immunity are being investigated in preclinical and ongoing clinical trials in ovarian cancer and lung cancer $[20,21]$. This led to our hypothesis that TNBC could be effectively targeted with immune-based cancer treatment using CAR T cells, an approach that has shown great utility on the treatment of hematological malignancy [22]. CAR T cell therapy represents a rapid approach to generate and apply a large number of antigenspecific $\mathrm{T}$ cells for the treatment of cancer, and in previous studies, we developed FR $\alpha$-specific CAR T cells that demonstrated potent effects on human ovarian cancer in preclinical models $[9,10]$. We therefore sought to utilize FR $\alpha$ CAR T cells to target TNBC.
Consistent with IHC assay results reported by others $[4,17,18]$, we found positive surface expression of FR $\alpha$ protein on all (5/5) human TNBC cell lines tested by flow cytometry. FR $\alpha$ expression was largely moderate on these TNBC lines; however, IHC findings demonstrate that FR $\alpha$ protein can be expressed at high, intermediate, or low levels in TNBC, and as with primary breast tumors, the abundance of FR $\alpha$ mRNA varies among TNBC cell lines, with a subset expressing high levels [4]. As we showed earlier, FR $\alpha$-specific CAR T cells are sensitive in vitro to FR $\alpha$ protein displayed on the cancer cell surface at a wide range of levels $[9,10]$. In the current study, primary human T cells expressing a FR $\alpha$-specific CAR recognized all human TNBC cell lines expressing FR $\alpha$ at different levels. $\mathrm{FR} \alpha$-specific $\mathrm{T}$ cells exhibited polyfunctionality in their ability to secrete IFN- $\gamma$, TNF- $\alpha$, and IL-2 upon stimulation with FR $\alpha+$ tumor cells. FR $\alpha$ CAR T cells also displayed potent cytolytic capacity in vitro against $\mathrm{FR} \alpha+\mathrm{TNBC}$ cell lines. These in vitro tumor killing findings further support 
the notion that FR $\alpha$ has promise as a novel immunotherapy target for TNBC, which currently lacks effective targeted therapy. Importantly, two injections of FR $\alpha$ CAR $T$ cells exhibited in vivo antitumor effects in a highly invasive and metastatic MDA-MB-231 xenograft model of human TNBC. Given the natural range of FR $\alpha$ protein expression level in TNBC, we postulated that the response to CAR T cell therapy may be more pronounced in patients with tumors expressing higher levels of FR $\alpha$. Indeed, our preclinical studies show that the antitumor activity of FR $\alpha$ CAR T cells correlates with the level of surface antigen expressed by tumor cells, as demonstrated by the more dramatic tumor regressions mediated by CAR $\mathrm{T}$ cells in mice bearing MDA-231 TNBC tumors in which FR $\alpha$ was expressed at a high level.

Our results rationalize the application of FR $\alpha$ CAR T cell therapy in patients with TNBC whose tumors express $\mathrm{FR} \alpha$, particularly at a high level. It is notable that the FR $\alpha$ gene is positively regulated by the glucocorticoid receptor agonist, dexamethasone, at the transcriptional (P4 promoter) level and this profound regulation is further potentiated by inhibiting histone deacetylase (HDAC) [23]. This observation supports the notion that the efficacy of FR $\alpha$ CAR T cell therapy in TNBC patients with low to intermediate tumoral FR levels may be improved in combination with dexamethasone and HDAC inhibitors that enhance FR $\alpha$ gene expression; however, additional preclinical studies of cell surface expression of FR $\alpha$ would be required to confirm this finding in vitro and in vivo, as well as their effects on normal tissue organ expression.

As an alternative approach to improve CAR $\mathrm{T}$ cell therapy for TNBC, simultaneous co-targeting of two different tumor-associated antigens may be applied to broaden the immune response and induce tumor elimination. For instance, like $\mathrm{FR} \alpha$, mesothelin, another GPI-linked cell surface glycoprotein present on mesothelial cells, is overexpressed in TNBC and has been shown to be an attractive immunotherapy target for CAR T cells [24]. We previously constructed a fully human anti-mesothelin CAR and showed that CAR-redirected T cells efficiently kill mesothelin-expressing tumors in vitro and in vivo [25]. Therefore, co-administration of FR $\alpha$ CAR $\mathrm{T}$ cells and mesothelin CAR $\mathrm{T}$ cells may provide superior antitumor effects in TNBC and also address potential issues of tumor antigen heterogeneity and antigen loss, which has been previously reported $[26,27]$. In this line, we have previously established proof of principle for the application of bispecific CAR T cells with specificity for both mesothelin and FR $\alpha$ [28]. However, Anurathapan et al. [29] demonstrated optimal antitumor effects using two different antigenspecific CARs simultaneously, though this was insufficient to achieve a complete response. Small numbers of residual tumor cells were observed that appeared to express the targeted antigens and were resistant to repetitive $\mathrm{T}$ cell treatment, likely due to the low level of antigen expression determining tumor susceptibility to CAR T cells. Similarly, in a multicenter trial [30] of a novel FR $\alpha$-targeted agent (EC145) in advanced, FR $\alpha$-positive adenocarcinoma of the lung, there was an overall survival advantage and superior clinical benefit response for patients who had high expression of FR $\alpha$ on their tumors compared with those who had tumors with intermediate FR $\alpha$ expression. Based on these results, a phase II trial (clinicaltrials.gov/NCT01577654) evaluating the activity of EC145 versus EC145 plus docetaxel versus docetaxel alone in FR $\alpha$ high tumors has been initiated. Collectively, results from these studies suggest that pre-selection of patients with a high-level antigen expression could improve clinical response rates.

In the clinic, another $\mathrm{T}$ cell-based immunotherapy approach utilizing bispecific $\mathrm{T}$ cell engager (BiTE) is also under investigation [31,32]. BiTEs provide a conventional drug approach in terms of storage, dosage, and delivery system; however, BiTEs have a short half-life and have to be given as a continuous infusion, which can be associated with systemic toxicities [33]. In addition, BiTEs lack active biodistribution once infused and may not penetrate tissue planes [33]. Compared with the BiTE approach, CAR T cell therapy has several advantages for TNBC and other cancers. CAR T cells can recognize and lyse cells bearing a very low level of target antigen, and CAR T cells can persist long term in vivo. Since our human FR $\alpha$ CAR T cells do not cross-react against mouse FR $\alpha$ protein, the potential for toxicity of this approach in the TNBC mouse model cannot be determined. Importantly, however, various strategies are being developed to create safer, regulatable CARs that control and/or minimize potential toxicity $[34,35]$.

While increased expression of FR $\alpha$ is associated with a poor clinical outcome in TNBC [17], FR $\alpha$ expression may vary by TNBC subtype and be associated with disease stage. Future studies will be required to determine the minimal and maximal threshold of FR $\alpha$ expression for activation and effective lysis by FR $\alpha$ CAR T cells upon stimulation with the TNBC cell lines or autologous tumor cells. Such results might aid in determining which patients may best benefit from FR $\alpha$ CAR T cell therapy and help determine the potential for offtumor targeting of healthy tissues that may express low levels of antigen.

\section{Conclusion}

We report the first demonstration of an in vivo antitumor response against established human TNBCxenografts using FR $\alpha$-redirected CAR T cell therapy, with increased sensitivity observed against tumors bearing higher FR $\alpha$ protein levels. We therefore conlude that 
pateints with TNBC that expresses a high level of FR $\alpha$ protein may benefit from $F R \alpha$-redirected CAR $T$ cell therapy. More so, our preclinical studies serve to further accelerate the translation of FR $\alpha$-targeted immunotherapies, including FR $\alpha$ CAR T cell therapy, to the clinic for TNBC.

\section{Additional files}

Additional file 1: Figure S1. Expression of FRa in enzymatically digested MDA-231 and cell lines and correlation with IFN- $\gamma$ secretion. FRa expression was determined using BD Quanti-Brite beads. The number of receptors per cell was calculated used at a 1:1 PE/protein ratio for the quantitative analysis of surface FRa expression. FRa antigen number per tumor cell was calculated by comparing the mean fluorescence intensity (MFI) versus the number of known PE molecules per bead. T47D and MDA-231 exhibited the highest number of FRa receptors per cell and AE17 mouse mesothelioma cell line displaying the lowest number of receptors per cell (A). Using a linear regression fitted line, the correlation between the Fra receptors per cell and IFN- $\gamma$ secretion was calculated (B). (TIF $51 \mathrm{~kb}$ )

Additional file 2: Figure S2. MDA-231 tumors retained a stable FRa expression profile after UNT, CD19 CAR, or FRa CAR T cell treatment. On day 73 , mice bearing MDA-231 tumors were sacrificed and tumors were collected and cut up in RPMI 1640, washed, and centrifuged at room temperature at $800 \mathrm{rpm}$ for $5 \mathrm{~min}$ and then resuspended in enzymatic digestion buffer (collagenase $[0.2 \mathrm{mg} / \mathrm{mL}$ ] and DNase [30 units/mL] in RPMI 1640) for overnight digestion at room temperature. FRa-specific mAb MOv18 was used to measure FRa expression on MDA-231 tumors treated with different T cells. (TIF $51 \mathrm{~kb}$ )

Additional file 3: Figure S3. FRa CAR T cells induced SKOV3 ovarian tumor rapid regression in vivo. NSG mice were inoculated with SKOV3 ovarian cancer tumor cells. Mice bearing established SKOV3 tumors received tail vein injections of $10^{7}$ CAR+ T cells on days 40 and 46 and tumor growth was monitored by caliper measurements. (TIF $140 \mathrm{~kb}$ )

Additional file 4: Figure S4. Tumor volume fold changes after CAR T cell treatment on days 60 and 74. NSG mice were inoculated with MDA-231 or MDA-231. FRa tumor cells. Mice bearing established MDA-231.FRa or MDA-231 tumors received tail vein injections of $1 \times 10^{7}$ CAR+ T cells on days 40 and 46 , and tumor growth was monitored by caliper measurements. (TIF $37 \mathrm{~kb}$ )

\section{Acknowledgements}

The authors gratefully acknowledge Drs. Lin Zhang, Qihong Huang, and Laura Barrio Real for providing the TNBC cell lines.

\section{Funding}

This work was supported by grants from the Basser Center for BRCA Research (DJP) for breast cancer studies, the Ovarian Cancer Research Foundation (DJP) for CAR T cell construction, and a generous gift from the Bethesda Foundation for in vitro assays.

\section{Availability of data and materials}

Due to our internal policy and those governing an alliance between Novartis and the University of Pennsylvania on CAR T cells in oncology, the raw data cannot be shared.

\section{Authors' contributions}

DJP and DS initiated and designed the in vitro and in vivo studies. DS and MP performed the preclinical animal studies. DS, OY, and JAC performed the in vitro experiments. MF aided in the construction of the vectors. DS, JAC, and DJP prepared the manuscript. All authors read and approved the final manuscript.

\section{Competing interests}

DJP holds patents in the area of CAR T cell therapy for oncology. The remaining authors declare that they have no competing interests.
Consent for publication

Not applicable.

\section{Ethics approval and consent to participate}

All animals were obtained from the Stem Cell and Xenograft Core (SCXC) of the Abramson Cancer Center, University of Pennsylvania. Mice were bred, treated, and maintained under pathogen-free conditions in-house under University of Pennsylvania IACUC-approved protocols and as mandated by federal law and regulations. The experimental protocol was conducted in accordance with the recommendations of the Guide for Care and Use of Laboratory Animals with respect to restraint, husbandry, surgical procedures, feed and fluid regulation, and veterinary care. The animal care and use program at the University of Pennsylvania is accredited by the Association for Assessment and Accreditation of Laboratory Animal Care International (AAALAC).

\section{Author details}

'Ovarian Cancer Research Center, Department of Obstetrics and Gynecology, Perelman School of Medicine, University of Pennsylvania, 3400 Civic Center Blvd, Rm 8-103 Smilow CTR, Philadelphia, PA 19104, USA. ${ }^{2}$ Department of Experimental Oncology and Molecular Medicine, Fondazione IRCCS Istituto Nazionale dei Tumori, Milan, Italy. ${ }^{3}$ Department of Pathology and Laboratory Medicine, Abramson Cancer Center, Perelman School of Medicine, University of Pennsylvania, 3400 Civic Center Blvd, Rm 8-103 Smilow CTR, Philadelphia, PA 19104, USA. ${ }^{4}$ Current address: Intrexon Corporation, 20358 Seneca Meadows Pkwy, Germantown, MD 20876, USA.

Received: 17 March 2016 Accepted: 7 July 2016

Published online: 20 July 2016

\section{References}

1. Foulkes WD, Smith IE, Reis-Filho JS. Triple-negative breast cancer. N Engl J Med. 2010;363(20):1938-48.

2. Tan DS, et al. Triple negative breast cancer: molecular profiling and prognostic impact in adjuvant anthracycline-treated patients. Breast Cancer Res Treat. 2008:111(1):27-44.

3. Cleator S, Heller W, Coombes RC. Triple-negative breast cancer: therapeutic options. Lancet Oncol. 2007:8(3):235-44.

4. O'Shannessy DJ, et al. Folate receptor alpha (FRA) expression in breast cancer: identification of a new molecular subtype and association with triple negative disease. Springerplus. 2012;1(1):1-9.

5. Rochman H, Selhub J, Karrison T. Folate binding protein and the estrogen receptor in breast cancer. Cancer Detect Prev. 1985;8(1-2):71.

6. Kelley KM, Rowan BG, Ratnam M. Modulation of the folate receptor a gene by the estrogen receptor mechanism and implications in tumor targeting. Cancer Res. 2003:63(11):2820-8.

7. Xia W, Low PS. Folate-targeted therapies for cancer. J Med Chem. 2010; 53(19):6811-24.

8. Clifton GT, et al. Folate receptor $a$ : a storied past and promising future in immunotherapy. Human Vaccines. 2011;7(2):183-90.

9. Song D-G, et al. CD27 costimulation augments the survival and antitumor activity of redirected human T cells in vivo. Blood. 2012;119(3):696-706.

10. Song D-G, et al. In vivo persistence, tumor localization, and antitumor activity of CAR-engineered $T$ cells is enhanced by costimulatory signaling through CD137 (4-1BB). Cancer Res. 2011;71(13):4617-27.

11. Milone MC, et al. Chimeric receptors containing CD137 signal transduction domains mediate enhanced survival of T cells and increased antileukemic efficacy in vivo. Mol Ther. 2009;17(8):1453-64.

12. Parry RV, et al. CD28 and inducible costimulatory protein Src homology 2 binding domains show distinct regulation of phosphatidylinositol 3-kinase $\mathrm{BCl}-\mathrm{xL}$, and IL-2 expression in primary human CD4 T lymphocytes. J Immunol. 2003;171(1):166-74

13. Figini $\mathrm{M}$, et al. Conversion of murine antibodies to human antibodies and their optimization for ovarian cancer therapy targeted to the folate receptor. Cancer Immunol Immunother. 2009;58(4):531-46.

14. Wolfl M, et al. Activation-induced expression of CD137 permits detection, isolation, and expansion of the full repertoire of CD8+ T cells responding to antigen without requiring knowledge of epitope specificities. Blood. 2007; 110(1):201-10 
15. Siu MK, et al. Paradoxical impact of two folate receptors, FRa and RFC, in ovarian cancer: effect on cell proliferation, invasion and clinical outcome. PLoS One. 2012;7(11):e47201.

16. Necela BM, et al. Folate receptor-a (FOLR1) expression and function in triple negative tumors. PLoS One. 2015;10(3):e0122209.

17. Hartmann LC, et al. Folate receptor overexpression is associated with poor outcome in breast cancer. Int J Cancer. 2007;121(5):938-42.

18. Zhang Z. et al. Folate receptor a associated With triple-negative breast cancer and poor prognosis. Arch Pathol Lab Med. 2013.

19. Knutson $\mathrm{KL}$, et al. T-cell immunity to the folate receptor alpha is prevalent in women with breast or ovarian cancer. J Clin Oncol. 2006:24(26):4254-61.

20. Konner JA, et al. Farletuzumab, a humanized monoclonal antibody against folate receptor $\mathrm{a}$, in epithelial ovarian cancer: a phase I study. Clin Cancer Res. 2010;16(21):5288-95.

21. Thomas A, Maltzman J, Hassan R. Farletuzumab in lung cancer. Lung Cancer. 2013;80(1):15-8.

22. Maude SL, et al. Chimeric antigen receptor T cells for sustained remissions in leukemia. N Engl J Med. 2014;371(16):1507-17.

23. Tran $\mathrm{T}$, et al. Enhancement of folate receptor a expression in tumor cells through the glucocorticoid receptor: a promising means to improved tumor detection and targeting. Cancer Res. 2005;65(10):4431-41.

24. Tchou J, et al. Mesothelin, a novel immunotherapy target for triple negative breast cancer. Breast Cancer Res Treat. 2012;133(2):799-804.

25. Lanitis $\mathrm{E}$, et al. Redirected antitumor activity of primary human lymphocytes transduced with a fully human anti-mesothelin chimeric receptor. Mol Ther. 2012;20(3):633-43.

26. Riker $\mathrm{A}$, et al. Immune selection after antigen-specific immunotherapy of melanoma. Surgery. 1999;126(2):112-20.

27. Grupp SA, et al. Chimeric antigen receptor-modified T cells for acute lymphoid leukemia. N Engl J Med. 2013;368(16):1509-18.

28. Lanitis $\mathrm{E}$, et al. Chimeric antigen receptor $\mathrm{T}$ cells with dissociated signaling domains exhibit focused antitumor activity with reduced potential for toxicity in vivo. Cancer Immunol Res. 2013;1(1):43-53.

29. Anurathapan $U$ et al. Kinetics of tumor destruction by chimeric antigen receptor-modified T cells. Mol Ther. 2013.

30. Edelman MJ, et al. Multicenter trial of EC145 in advanced, folate-receptor positive adenocarcinoma of the lung. J Thorac Oncol. 2012;7(10):1618-21.

31. Wu J, et al. Blinatumomab: a bispecific T cell engager (BiTE) antibody against CD19/CD3 for refractory acute lymphoid leukemia. J Hematol Oncol. 2015;8(1):1.

32. Fan G, et al. Bispecific antibodies and their applications. J Hematol Oncol. 2015;8(1):1.

33. Stone JD, et al. A sensitivity scale for targeting $T$ cells with chimeric antigen receptors (CARs) and bispecific T-cell engagers (BiTEs). Oncoimmunology. 2012;1(6):863-73.

34. Rodgers DT, et al. Switch-mediated activation and retargeting of CAR-T cells for B-cell malignancies. Proc Natl Acad Sci. 2016;113(4):E459-68.

35. Wu CY, et al. Remote control of therapeutic $T$ cells through a small molecule-gated chimeric receptor. Science. 2015;350(6258):aab4077.

\section{Submit your next manuscript to BioMed Central and we will help you at every step:}

- We accept pre-submission inquiries

- Our selector tool helps you to find the most relevant journal

- We provide round the clock customer support

- Convenient online submission

- Thorough peer review

- Inclusion in PubMed and all major indexing services

- Maximum visibility for your research

Submit your manuscript at www.biomedcentral.com/submit

) Biomed Central 